\title{
A Surface Plasmon Resonance Histamine Sensor Based on an Electropolymerized Molecularly Imprinted Polymer (E-MIP)
}

\author{
Frances Adeline D. Hubilla, Arvin I.Mabilangan, Rigoberto C. Advincula and Florian R. del Mundo \\ Institute of Chemistry, University of the Philippines - Diliman, Quezon City, 1101 Philippines
}

\begin{abstract}
In this paper, histamine sensing by surface plasmon resonance (SPR) spectroscopy using an electropolymerized molecularly imprinted polymer (E-MIP) of functionalized polyterthiophene and histamine is reported. The MIP histamine sensor showed good linear relationship $\left(\mathrm{R}^{2}=0.995\right)$ between the SPR angle shift and the logarithm of histamine concentration, ranging from $15-500 \mu \mathrm{g} / \mathrm{mL}$, with a limit of detection (LOD) of $2.0 \mu \mathrm{g} / \mathrm{mL}$ and a calibration sensitivity of $44.6 \mathrm{~m}^{\circ}$ per $\log \mu \mathrm{g} / \mathrm{mL}$ histamine. Results also showed higher binding affinity of histamine for the MIP sensor than for the non-imprinted (NIP) sensor indicating excellent specificity. Moreover, the selectivity of the MIP histamine sensor against some of its structural analogues was demonstrated.
\end{abstract}

\section{Introduction}

Histamine is a by-product of spoilage and a good indicator of temperature abuse and state of good manufacturing practices adopted in the handling of fish/shrimp harvests. The World Health Organization (WHO) standard for good hygienic practices (GHP) and hazard analysis critical control point (HACCP) as applied to storing fish and shrimp products required that the level of histamine must be lower than $15 \mathrm{ppm}(\mathrm{mg} / \mathrm{kg})$ [1].

Conventional methods for histamine detection include HPLC [2], spectrofluorometry [3], colorimetry [4], ELISA [5], and the widely-accepted AOAC fluorometric method [6]. These methods have relatively low limits of detection for histamine, but require expensive instrument and tedious sample pretreatment to eliminate potential interference from other imidazole compounds such as histidine.

Molecular imprinting technology involves the fabrication of materials called molecularly imprinted polymers (MIPs), which are capable of recognizing target molecules with high selectivity and affinity [7]. From the interaction of the functional monomer polymerized around the template molecule, a highly cross-linked polymer complex will be formed. Removing the template molecule will produce an imprint of it on the polymer film and recognition sites for the template molecule will be available.

Surface plasmon resonance spectroscopy is a technique based on the interaction of surface plasmons- a charge density wave which occurs at the interface of a metal and a dielectric, and incident light. The incident electromagnetic wave, when in resonance with the plasmons, leads to collective oscillations, which is observed as a sharp dip in the reflected light intensity of the spectrum (Fig. 1a and 1b).

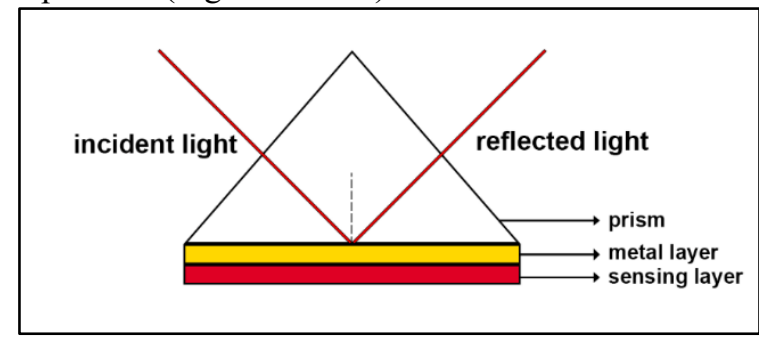

(a)

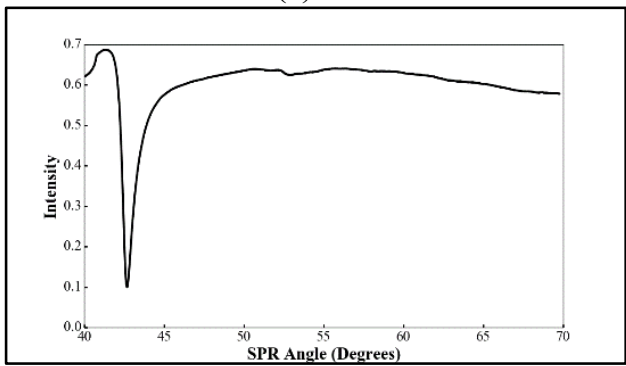

(b)

Figure 1. Kretschman configuration of SPR and b. An SPR curve showing the sharp dip caused by excitation of the surface plasmons.

Beyond the metal surface is the evanescent electric field. This field interacts with the area around the metal, which in this study is the area around the immobilized MIP. Every time there is a change in the evanescent region (e.g. presence of analytes interacting with the MIP), the SPR angle changes. This is the basis of SPR for biosensing applications [8].

The development of SPR-based sensors has been applied to a variety of fields over the recent years as they present an alternative in detection as compared to 
conventional techniques [9]. Not only they can offer fast, sensitive, and on-site analysis, SPR sensors have also made significant contributions in high-throughput screening used in numerous important applications such as medical diagnostics, environmental monitoring, food safety, and security [10].

In this paper, the development of a surface plasmon resonance (SPR) histamine sensor using ultrathin films of electropolymerized molecularly imprinted polymer (EMIP) of functionalized polyterthiophene and histamine is reported.

\section{Experimental}

\subsection{Chemical reagents}

Histamine (freebase), tetrabutylammonioumhexafluorophosphate (TBAH) ,cadaverine, putrescine, and Lhistidine were purchased from Sigma-Aldrich (Missouri, USA). Acetonitrile (HPLC grade) and sodium hydroxide pellets (ACS reagent) were purchased from J.T. Baker. The terthiophene monomer (Sigma-Aldrich, Missouri) was functionalized with carboxylic acid $(\mathrm{G} 03 \mathrm{TCOOH})$ prior to use [11].

\subsection{MIP fabrication}

The optimum histamine:G03TCOOH ratio was predetermined using modelling studies via the semiempirical PM3 calculations of the Orca program. All solids were dissolved with 0.1 M TBAH in 80:20 ACN: $\mathrm{H}_{2} \mathrm{O}$ as supporting electrolyte.

The pre-polymer complex solution was electropolymerized by cyclic voltammetry in a standard three-electrode measuring cell using an Ivium Technologies CompactStat potentiostat. The reference electrode is an $\mathrm{Ag} / \mathrm{AgCl}$ electrode, the counter electrode is a platinum rod electrode and the working electrode is a Bionavis Ltd SPR gold sensor slide (Au-coated BK7 glass slides, 12 × $20 \mathrm{~mm}$ ). An NIP was also fabricated in the absence of the template molecule to act as the control. The imprinted template molecule was extracted using $0.01 \mathrm{M} \mathrm{NaOH}$ in 80:20 ACN: $\mathrm{H}_{2} \mathrm{O}$.

\subsection{Performance evaluation of the fabricated E- MIP histamine sensor}

SPR measurements were performed with SPR NaviTM 200 (BioNavis Ltd, Finland). The SPR response of the fabricated E-MIP sensor to standard histamine solutions was evaluated from 0-500 $\mu \mathrm{g} / \mathrm{mL}$. All histamine standard solutions were prepared in 75:25 MeOH: $\mathrm{H}_{2} \mathrm{O}$. From the generated standard calibration plot of SPR angle shift versus the logarithm of the histamine concentration $(\mu \mathrm{g} / \mathrm{mL})$, the linear concentration range, calibration sensitivity, LOD, and limit of quantitation (LOQ) were calculated. The MIP SPR responses to histamine and its structural analogues were also compared.

\section{Results and discussion}

\subsection{MIP fabrication}

Histamine interacts with G03TCOOH through noncovalent interaction in the aliphatic amine group of histamine. The molecular ratio between histamine and G03TCOOH was optimized through modelling studies and was found to be 1:3 (histamine:G03TCOOH), shown in Fig. $2 a$ and $2 b$.

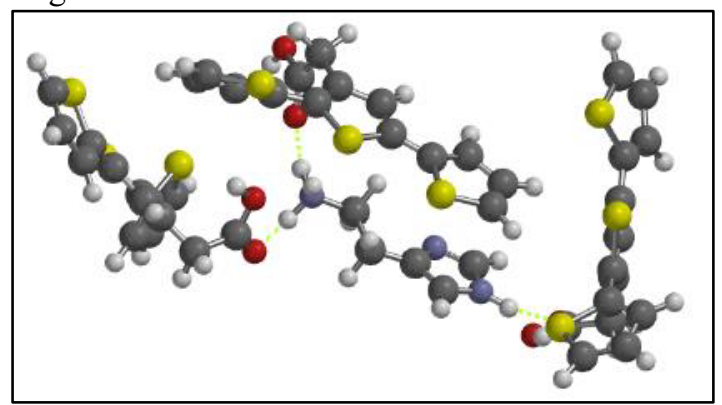

(a)

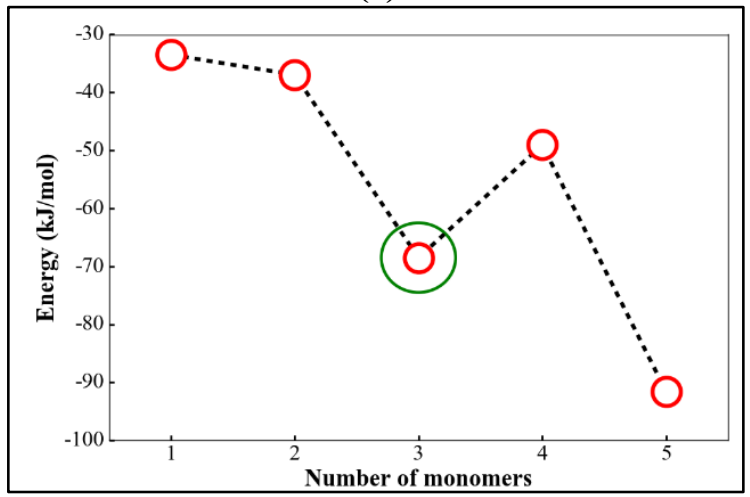

(b)

Figure 2. optimized simulated structure of histamine complexed with three G03TCOOH molecules and b. calculated stabilizing interaction in histamine-G03TCOOH complexes. Encircled point indicates the number of G03TCOOH monomers which gives the first and most stable arrangement with histamine.

Fig. 3 illustrates the SPR curves of the MIP at different stages of fabrication (gold slide only, electropolymerization, template removal, rebinding). A noticeable shift in the SPR angle will be observed due to changes in the dielectric constant of the film layer upon interaction of the template and the MIP sensor [12].

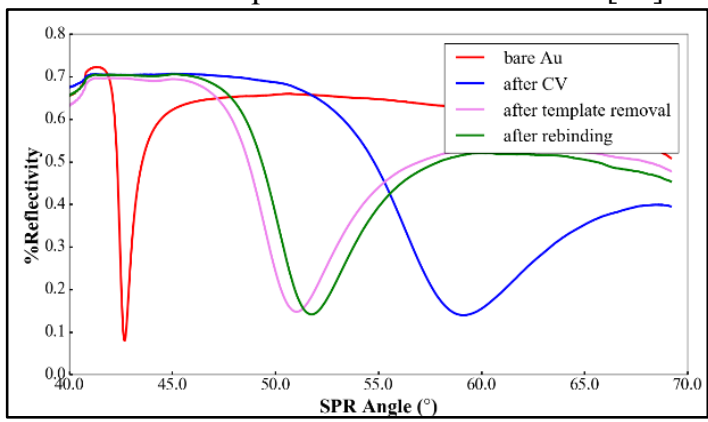

Figure 3. SPR curves of one MIP sensor at different fabrication stages.

Using the pre-determined template monomer ratio of $1: 3$, the sensor was electropolymerized using cyclic voltammetry. The number of cycles and scan rate were optimized as these affect the thickness of the sensor. 
For the extraction of histamine from the electropolymerized polymer complex, a basic solution containing $\mathrm{NaOH}$ was used. The base deprotonates the aliphatic amine group of histamine, dissociating the complex and releasing histamine into the solution [13].

SPR was used to monitor the template removal time by obtaining the SPR curves at certain time intervals. A plot of angle shift versus time is shown in Fig. 4.

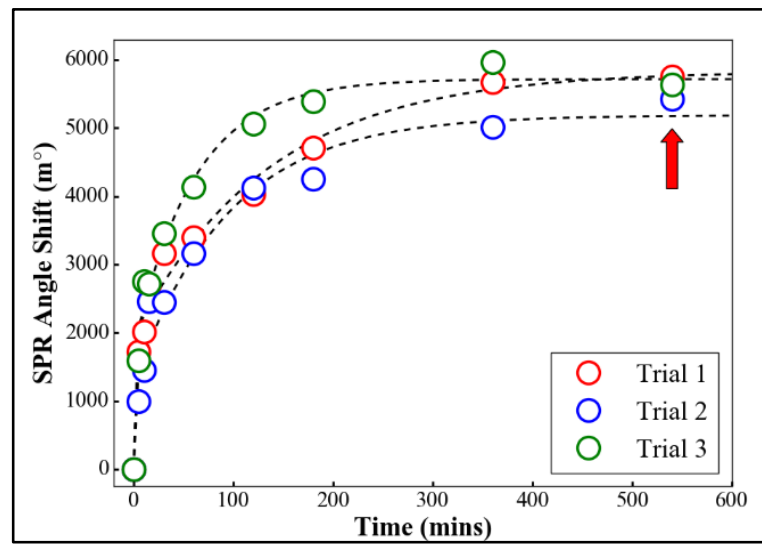

Figure 4. SPR angle shift versus template removal time using $0.01 \mathrm{M} \mathrm{NaOH}$ in $80: 20 \mathrm{ACN}: \mathrm{H}_{2} \mathrm{O}$ for $1 \mathrm{mM}$ histamine: $3 \mathrm{mM}$ terthiophene MIP sensor.

Optimum time was chosen when the SPR angle shift is less than 10 millidegrees per 5 minutes. As shown in Fig. 4, at the optimum time of nine hours, it is assumed that the $1 \mathrm{mM}$ histamine template has been completely extracted from the polymer, hence no changes in the optical thickness (angle shift) can be observed.

Rebinding studies on the extracted histamine were also performed using SPR. The time it takes for the MIP sensor to reach equilibrium response upon rebinding was determined. The sensor was equilibrated with $1 \mathrm{mM}$ histamine in 75:25 MeOH: $\mathrm{H}_{2} \mathrm{O}$ at different time intervals. SPR readings were taken at the end of each time interval and a plot of SPR angle shift at each time interval was made as shown in Fig. 5.

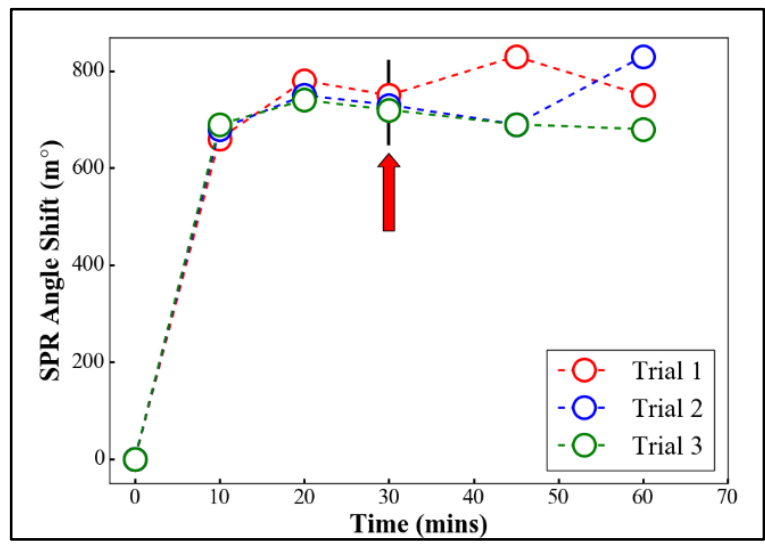

Figure 5. Rebinding time optimization for the MIP sensor. Concentration of the injected histamine solution is $1 \mathrm{mM}$ in 75:25 MeOH: $\mathrm{H}_{2} \mathrm{O}$.

At the plateau portion of the plot, no significant change in the optical thickness (angle shift) was observed. The optimum equilibrium rebinding time of 30 minutes was selected where the SPR angle shift is less than 10 millidegrees per 5 minutes. At this point the MIP sensor has been completely saturated with the injected histamine molecules. This optimum rebinding time was used for the succeeding rebinding experiments, including calibration and selectivity studies.

To check if histamine was indeed imprinted on the MIP sensor, rebinding studies were also performed on the NIP. Injection of histamine in the NIP should show no significant response as it does not have the binding sites for histamine. The plot in Fig. 6 showed that the NIP exhibited no significant response upon injection of histamine at different concentrations compared to the MIP. The slight increase in response in the NIP however, may be attributed to the nonspecific binding of histamine to the NIP.

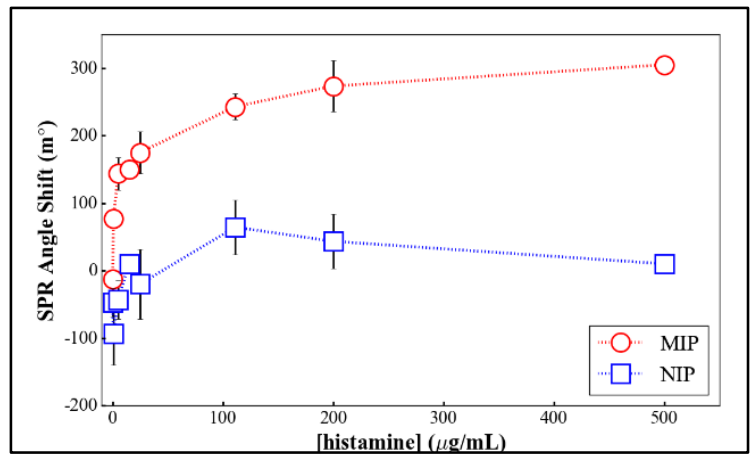

Figire 6. Binding of histamine on the MIP and the NIP. Error bars represent standard deviation of 3 trials.

\subsection{Evaluation of the analytical performance of the histamine MIP sensor}

The generated response curve of SPR angle shift versus histamine concentration from $0-500 \mu \mathrm{g} / \mathrm{L}$ is shown in Fig. 7.

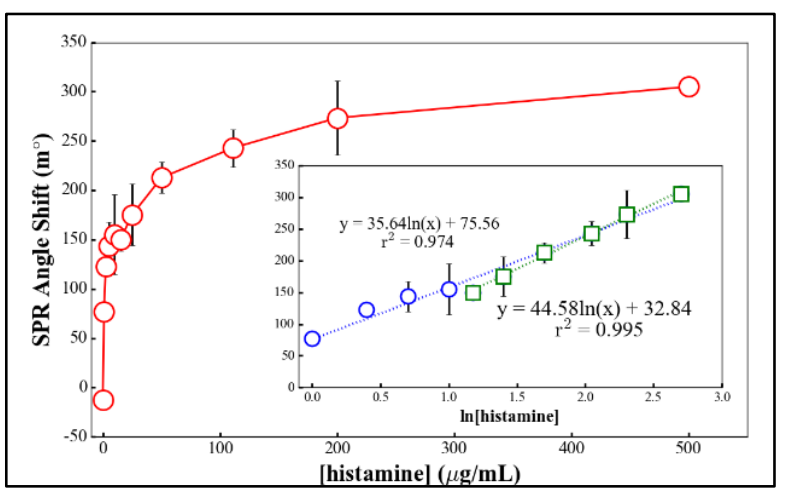

Figure 7. Response curve and standard calibration plot for the histamine MIP sensor. Squares correspond to the linear range.

The response curve is similar to a typical binding isotherm with an initial sharp rise followed by gradual saturation. The standard calibration plot (Fig. 7 inset) of the SPR angle shift versus log histamine concentration $(\mu \mathrm{g} / \mathrm{mL})$ showed good linear relationship $\left(\mathrm{R}^{2}=0.995\right)$ from $15-500 \mu \mathrm{g} / \mathrm{mL}$. The LOD and LOQ of the sensor were calculated using the following equations:

$$
\begin{aligned}
& \mathrm{LOD}=\frac{3 \sigma_{\text {blank }}}{m} \\
& \mathrm{LOQ}=\frac{10 \sigma_{\text {blank }}}{m}
\end{aligned}
$$


In equations (1) and (2), $\sigma_{\text {blank }}$ refers to the standard deviation of the blank readings and $m$ corresponds to the slope of the standard calibration curve. The obtained LOD and LOQ of the sensor are $2.0 \mu \mathrm{g} / \mathrm{mL}$ and 12 $\mu \mathrm{g} / \mathrm{mL}$, respectively. The calibration sensitivity is equal to $44.6 \mathrm{~m}^{\circ}$ per $\log \mu \mathrm{g} / \mathrm{mL}$ histamine.

Fig. 8 shows the relative response of the the MIP sensor to histamine structural analogues. The MIP sensor showed no response to L-histidine, and relatively lower responses to cadaverine and putrescine compared to histamine indicating high selectivity of the fabricated MIP sensor to histamine. The possibility that most of the binding sites in the MIP complements the aliphatic amine group of histamine might account for the increasing trend found in the response of the MIP to cadaverine and putrescine, as these two analogues also have aliphatic amines in their structure.

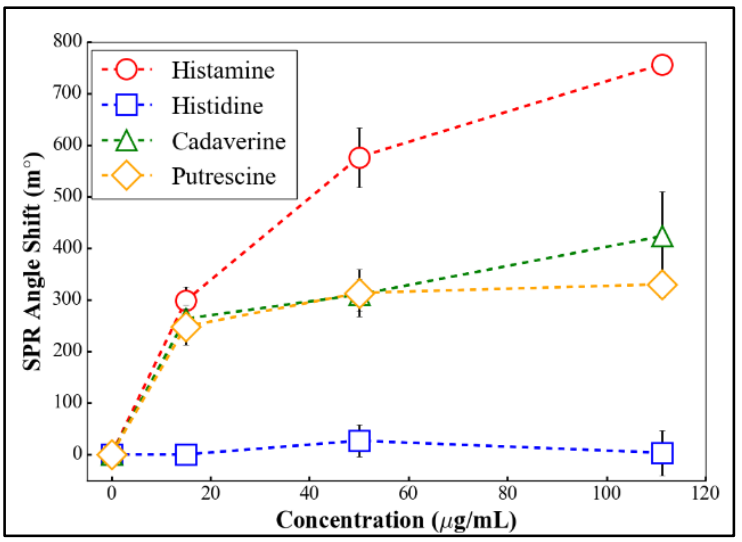

Figure 8. Selectivity (relative response) of the MIP sensor to histamine compared to L-histidine, cadaverine, and putrescine.

\section{Conclusions}

Detection and sensing of histamine was successfully achieved with the use of SPR spectroscopy and E-MIP of polyterthiophene and histamine. The analytical performance of the fabricated MIP sensor showed a good linear relationship $\left(\mathrm{R}^{2}=0.995\right)$ from $15-500 \mu \mathrm{g} / \mathrm{mL}$, an LOD of $2.0 \mu \mathrm{g} / \mathrm{mL}$ and a calibration sensitivity equal to $44.6 \mathrm{~m}^{\circ}$ per $\log \mu \mathrm{g} / \mathrm{mL}$ histamine. The MIP sensor also exhibited high selectivity for histamine versus various histamine analogues like L-histidine, cadaverine, and putrescine. The fabricated MIP histamine sensor has high potential for use as a quality assurance sensor to detect "freshness" of fish and shrimp harvests.

\section{Acknowledgement}

This work was supported by the Department of Science and Technology - Philippine Council for Industry, Energy, and Emerging Technology Research and Development (DOST-PCIEERD). The authors also would like to thank Dr. Rigoberto C. Advincula for the donated functionalized terthiophene monomer.

\section{References}

1. FAO/WHO (Food and Agriculture Organization of the United Nations/World Health Organization), Public Health Risks of Histamine and Other Biogenic Amines from Fish and Fishery Products Meeting Report (2013)

2. A. Sahebnasagh, G. Karimi, and S.A. Mohajeri: Food Anal. Methods, 7 (2014)

3. M.C. Vidal-Carou, M.L. Izquierdo-Pulido, A Mariné-Font: JAOAC, 72 (1989)

4. T. Kuda, T. Mihara, and T. Yano: Food Control, 18 (2007)

5. A. Marcobal, M.C. Polo, P.J. Martín-Álvarez, and M.V. Moreno-Arribas: Food Res. Int., 38 (2005)

6. AOAC (Association of Official Analytical Chemists), JAOAC, 60 (1977)

7. W. Li and S. Li, Adv. Polym. Sci. 206 (2007)

8. M. Bera and M. Ray: Opt. Eng. 50 (2011)

9. H. Šípová, S. Zhang, A.M. Dudley, D. Galas, K. Wang, and J. Homola: Anal. Chem., 82 (2010)

10. D.C. Apodaca, R.B. Pernites, R. Ponnapati, F.R. del Mundo, and R.C. Advincula: Macromolecules, 44 (2011)

11. M. Piliarik, L. Párová, and J. Homola: Biosens. Bioelectron., 24 (2009)

12. P. Taranekar, T. Fulghum, A. Baba, D. Patton, and R.C. Advincula: Langmuir, 23 (2007).

13. A. Pietrzyk, S. Suriyanarayanan, W. Kutner, R. Chitta, and F. D’Souza, Anal. Chem., 81 (2009) 